\title{
Management of IEEE 802.11e Wireless LAN for Realtime QoS-Guaranteed Teleconference Service with Differentiated H.264 Video Transmission*
}

\author{
Soo-Yong Koo, Byung-Kil Kim, and Young-Tak Kim ${ }^{* *}$ \\ Dept. of Information and Communication Engineering, \\ Graduate School, Yeungnam University \\ 214-1, Dae-Dong, Kyungsan-Si, Kyungbook, 712-749, Korea \\ sykoo@tsc.ac.kr, woo_sue@hotmail.com, ytkimayu.ac.kr
}

\begin{abstract}
Various realtime multimedia applications will be provided in next generation Internet where IEEE 802.11e wireless LAN will be widely used as broadband access networks. The characteristics of the wireless channels in IEEE 802.11 (i.e., fluctuating bandwidth and large error rate), however, impose challenging problems in the efficient QoS-guaranteed realtime multimedia communications with strict QoS requirements (i.e., bandwidth, delay, jitter, and packet loss/error rate). In this paper we propose management schemes of IEEE 802.11e wireless LAN (WLAN) for realtime QoS-guaranteed teleconference services with differentiated H.264 video transmission. In the proposed scheme, the IEEE 802.11e Wireless LAN is managed to transmit I, P and B slices from H.264 encoder using different channels of both HCF controlled channel access (HCCA) and enhanced distributed channel access (EDCA). We compare several different mapping scenarios, and analyze the QoS provisioning performance for realtime multimedia teleconference service.
\end{abstract}

Keywords: IEEE 802.11e, H.264, QoS, Diffserv, HCCA, EDCA.

\section{Introduction}

End-to-end QoS-guaranteed differentiated service provisioning is essential in next generation Internet and Broadband convergence Network $(\mathrm{BcN})$ where various wired and wireless networks are interconnected. Especially, realtime multimedia services, such as VoIP and multimedia teleconference, require strict bandwidth and QoS requirements (i.e., committed data rate with given burst size, delay, jitter, and packet loss/error rate). The IEEE 802.11 wireless LAN [1-4] and IEEE 802.16 wireless MAN are expected to be used widely in next generation Internet as broadband wireless access network. The characteristics of the wireless channel in IEEE 802.11 (i.e., fluctuating bandwidth and large error rate), however, impose challenging

\footnotetext{
* This research was supported by the MIC, under the ITRC support program supervised by the IITA.

*** Corresponding author.
} 
problems in the efficient QoS-guaranteed realtime multimedia communications with strict QoS requirements.

H.264/AVC is the newest video coding standard that was approved by ITU-T as Recommendation H.264 and by ISO/IEC as International Standard 14496-10(MPEG4 part 10) Advanced Video Coding (AVC)[5-8]. H.264/AVC provides good video quality at broad range of bit rates and picture sizes, ranging from very low bit rate, low frame rate, postage stamp resolution video for mobile and dial-up devices, through to entertainment-quality standard definition(SD) and high definition(HD) TV. H.264/AVC also can be efficiently used in a resource limited environments such as WLAN. H.264 video encoder generates video stream in separate units of I (intra), P (predicted), and B (bi-predictive) slices according to the selected profile (baseline, main, extended) for different applications.

IEEE 802.11e [1] proposed new enhanced QoS provisioning mechanism that promises to ensure good QoS to applications depending upon its traffic category \& type. These two channel access functions are managed by a centralized controller called Hybrid Coordinator (HC) which is a module in the QoS Access Point (QAP).

Most research works for video transmission over IEEE 802.11e have been studied with EDCA mechanism[9]. In [9], where parameter set information (PSI), instantaneous decoding refresh (IDR) picture slice, partition $\mathrm{A}$, partition $\mathrm{B}$, partition $\mathrm{C}$ are transmitted through EDCA access category $\mathrm{AC} \_\mathrm{VO}, \mathrm{AC}$ _VI and AC_BE. The differentiated video transmission with delay constraints for teleconference on IEEE 802.11e WLAN with HCCA and EDCA, however, has not been fully studied yet. In this paper, we analyze the management of the IEEE 802.11e wireless LAN channels of HCCA and EDCA for QoS-guaranteed realtime multimedia teleconference service with differentiated H.264/AVC video transmission.

The rest of this paper is organized as follows. In section 2, we briefly explain the related work, such as H.264/AVC, IEEE 802.11e with HCCA and EDCA and transmission of H.264 video traffic over IEEE 802.11e. In section 3, we study various mapping scenarios of H.264/AVC video transmission on IEEE 802.11e HCCA \& EDCA channels. In section 4, we analyze the QoS provisioning performance of each mapping scenario, considering the end-to-end delay (less than $400 \mathrm{~ms}$ ) for multimedia teleconference. Finally we conclude in section 5.

\section{Background and Related Work}

\subsection{IEEE 802.11e Wireless LAN with HCCA and EDCA}

IEEE 802.11e [1] proposed new enhanced QoS provisioning mechanism that promises to ensure good QoS to applications depending upon its traffic category \& type. IEEE 802.11e MAC includes an additional coordination function called Hybrid Coordination Function (HCF). The HCF uses both a contention-based channel access method, called the enhanced distributed channel access (EDCA) mechanism for contention-based transfer, and a controlled channel access, referred to as the HCF controlled channel access (HCCA) mechanism, for contention-free transfer[1]. 
The QoS provisioning on IEEE 802.11e is based on enhanced distributed channel access by EDCA and centralized channel access by HCCA. These two channel access functions are managed by a centralized controller called Hybrid Coordinator (HC) which is a module in the QoS Access Point (QAP).

EDCA is the contention-based medium access method, and is realized with the introduction of traffic categories (TCs). The EDCA provides differentiated distributed access to the wireless medium for 8 priorities of stations. EDCA defines the access category $(\mathrm{AC})$ mechanism that provides support for the priorities at the stations. Each station may have up to 4 ACs (AC_VO, AC_VI, AC_BE and AC_BK) to support 8 user priorities (UPs). One or more UPs are assigned to one AC. Even though EDCA provides differentiated access categories, it does not guarantee the QoS parameters of hard realtime applications, i.e., jitter and delay.

In order to provide realtime services with guaranteed QoS-parameters, HCCA that has been designed for parameterized QoS support with contention-free polling-based channel access mechanism must be used. The QAP scheduler computes the duration of polled-TXOP (transmission opportunity) for each QSTA based upon the traffic specification (TSPEC) parameters of an application flow. The scheduler in each QSTA then allocates the TXOP for different traffic stream (TS) queues according to the priority order. In IEEE 802.11e, TSPEC is used to describe the traffic characteristics and the QoS requirements of a data flow to and from QSTA.

\subsection{H.264/AVC Standard}

H.264/AVC has been developed for higher compression of moving pictures for various applications such as videoconferencing, digital storage media, television broadcasting, Internet streaming, and communication [5]. It is also designed to enable the use of the coded video representation in a flexible manner for a wide variety of network environments. H.264/AVC introduces a set of error resiliency techniques such as slice structure, data partitioning (DP), flexible macroblock ordering (FMO), arbitrary slice ordering (ASO), and redundant pictures.

H.264/AVC is divided into two distinct layers. First, the video coding layer (VCL) is responsible for efficient representation of the video data based on motion compensation, transform coding, etc. Second, the network abstraction layer (NAL) is responsible for delivery over various types of network. H.264/AVC codec maps VCL data (a sequence of bit representing the coded video picture) into packets known as NAL units (NALUs) prior to transmission or storage. An NALU corresponds to a slice (or a parameter set). Each slice is to be packetized into its own RTP packet conforming to the RFC 3984 [12] packetization scheme. An NALU consists of a onebyte header and raw byte sequence payload (RBSP) that represents the MBs of a slice.

H.264/AVC defines three profiles, each supporting a particular set of coding functions for potential different applications. Baseline profile supports intra and intercoding (using I-slices and P-slices), and its potential applications include videotelephony, video-conference, and wireless communications. Main profile supports interlaced video, inter-coding using B-slices, inter-coding using weighted prediction and entropy coding, and its potential application includes television broadcasting and video storage. Extended profile does not support interlaced video, but adds modes to 
enable efficient switching between coded bit streams and improved error resilience (i.e. data partitioning), and is particularly useful for streaming media applications.

\subsection{Transmission of H.264 over IEEE 802.11e}

Adlen Ksentini et. al proposed a cross-layer architecture of H.264 video transmission over IEEE 802.11e WLAN that leverage the inherent H.264 error resilience tools and the existing QoS-based IEEE 802.11e MAC protocol possibilities [9]. In this crosslayer design, authors favor more interaction between the H.264's VCL that divides the original streams through data partitioning (DP) and the MAC that treats video streams with different EDCA access categories. NAL encapsulates slices generated by VCL to NALU. [9] focuses particularly on the nal_ref_idc (NRI) field in the NAL header. The NRI contains two bits that indicate the priority of the NALU payload, where 11 is the highest transport priority, followed by 10 , then by 01 , and finally, 00 is the lowest.

In the proposed architecture, the slices from the VCL have been differentiated according to the slice type (i.e., parameter set information, IDR picture, partition A, B and $\mathrm{C})$. EDCA access category $3\left(\mathrm{AC} \_\mathrm{VO}\right)$ is used to transmit parameter set information, while EDCA AC_VI is used to transmit IDR picture and partition A. EDCA AC_BE has been used to transmit partition $\mathrm{B}$ and partition $\mathrm{C}$. In this way, partition $\mathrm{B}$ and $\mathrm{C}$ are differentiated from background traffic (AC_BK).

In the proposed architecture, H.264 extended profile has been used with data partitioning option. The results obtained indicated that the proposed architecture achieves better performances in terms of delays and loss rate that the IEEE 802.11 DCF and 802.11e EDCA with single access category. The packet delays of IDR and partition A of the proposed architecture are shown to be less than $500 \mathrm{~ms}$; however, the packet delays of partition B and C are not explained, and the overall PSNR is not analyzed. Also, the proposed mapping algorithm uses only EDCA access categories to transmit H.264 packets. It may cause higher packet loss in high priority ACs and degradation of perceived video quality when network load becomes significant, or many nodes that transmit H.264 traffic compete with each other.

\section{H.264/AVC Video Transmission on IEEE 802.11e HCCA and EDCA Channels}

\subsection{Analysis of H.264/AVC Video}

In order to design an efficient mapping architecture of H.264/AVC video slices/ partitions and IEEE 802.11e HCCA/EDCA channels, we firstly analyzed the amount of traffic generated of each slice type, and the relationship between packet loss rate of each slice type and the peak signal to noise ratio (PSNR) in extended profile and baseline profile, respectively. For the analysis, Foreman and Paris CIF(352x288) video sequence have been used, these sample video clips were encoded using JM 10.2 reference software[13] with following encoder parameters setting: IDR picture period is 30 frames, frame rate is set constant at $30 \mathrm{fps}$, output file mode is RTP, and the slice size is 1500 bytes. 
Table 1. Composition of slice types in the results of H.264 encoding

(a) Case 1 - Extended profile

\begin{tabular}{|c|c|c|c|c|c|c|}
\cline { 3 - 7 } \multicolumn{2}{c|}{} & PSI & I & P & B & Total \\
\hline Foreman & percentage & $0.002 \%$ & $10.25 \%$ & $75.36 \%$ & $14.388 \%$ & $100 \%(394 \mathrm{kbps})$ \\
\cline { 2 - 7 }$(9.8 \mathrm{sec})$ & $\#$ of pkts & 2 & 36 & 320 & 149 & 507 \\
\hline \multirow{2}{*}{$\begin{array}{c}\text { Paris } \\
(35 \mathrm{sec})\end{array}$} & percentage & $0.002 \%$ & $20.008 \%$ & $66 \%$ & $13 \%$ & $100 \%(385 \mathrm{kbps})$ \\
\cline { 2 - 7 } & $\#$ of pkts & 2 & 238 & 1019 & 531 & 1790 \\
\hline
\end{tabular}

(b)Case 2 - Baseline profile

\begin{tabular}{|c|c|c|c|c|c|}
\cline { 2 - 5 } \multicolumn{2}{c|}{} & PSI & I & P & Total \\
\hline Foreman & percentage & $0.002 \%$ & $16.92 \%$ & $83.08 \%$ & $100 \%(474 \mathrm{kbps})$ \\
\cline { 2 - 6 }$(9.8 \mathrm{sec})$ & $\#$ of pkts & 2 & 73 & 470 & 545 \\
\hline $\begin{array}{c}\text { Paris } \\
(35 \mathrm{sec})\end{array}$ & percentage & $0.002 \%$ & $29.008 \%$ & $70 \%$ & $100 \%(503 \mathrm{kbps})$ \\
\cline { 2 - 6 } & $\#$ of pkts & 2 & 474 & 1543 & 2019 \\
\hline
\end{tabular}

Table 1 (a) and (b) show the composition of the slice types in H.264 encoding with extended profile and baseline profile, respectively. In extended profile, the total transmission rates are $394 \mathrm{kbps}$ for Foreman sequence and $385 \mathrm{kbps}$ for Paris sequence, where generated traffic of I slice is $10.25 \%$, P slices $75.36 \%$, and B slice $14.387 \%$ for Foreman sequence in extended profile. In baseline profile, only I and $\mathrm{P}$ slices are generated, and the ratio of PSI, I, and P slices for Foreman sequence are $0.002 \%, 16.92 \%$, and $83.08 \%$ respectively. The compositions of the slice types of Paris sequence have been analyzed to be in the same trend.

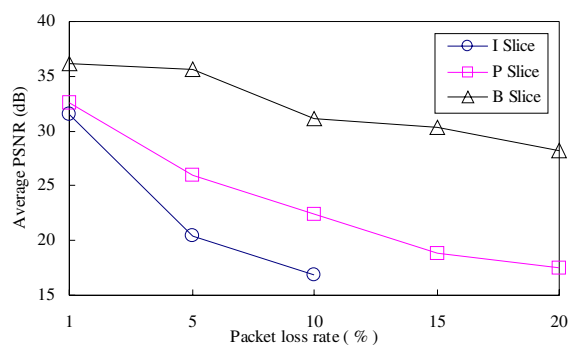

(a) Extended Profile

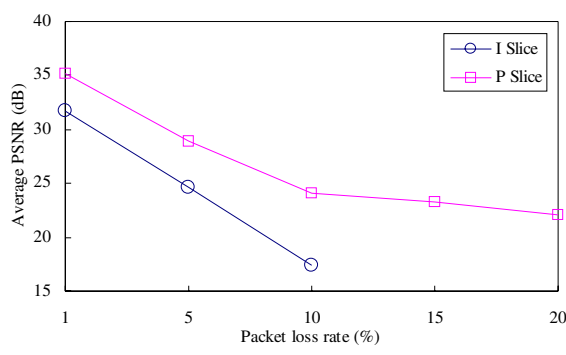

(b) Baseline Profile

Fig. 1. PSNR according to packet loss rate of each H.264 slice type

Fig. 1 depicts the relationship between packet loss rate of each H.264 slice type and the peak signal to noise ratio (PSNR) for Foreman sequence. Packet loss in I slice has the highest impact on the PSNR, P slice has the next impact, while B slice has the least impact on PSNR. This analysis results mean that I slice should be given the highest priority for least packet loss, while B slice can be given the lowest priority among the PSI, I, P and B slices from H.264 encoder. In the H.264 decoding simulation in ns-2, we found that when the PSNR is less than $30 \mathrm{~dB}$, the result video replay was at the status of annoying. So, the packet loss rate of I slices and P slices must be guaranteed to be less than $5 \%$. 


\subsection{Differentiated QoS-Provisioning with IEEE 802.11e HCCA and EDCA Channels}

Fig. 2. depicts a mapping scheme between DiffServ class-types and IEEE 802.11e HCCA and EDCA channels. Since the class-types of NCT, EF and AF4 require tight end-to-end packet delay and jitter bound, they are mapped onto HCCA; AF3/2/1 and $\mathrm{BEF}$ are mapped onto EDCA that can support flexible bandwidth usage with less stringent time constraints. By allocating HCCA channels, we can guarantee the bandwidth, while some EDCA channels may not guarantee bandwidth and delay if admission control mandatory (ACM) is not configured in lower priority channels.

In the differentiated transmission in IEEE 802.11e HCCA and EDCA channels, the delay, jitter, and available bandwidth guarantee are very important in the QoSguaranteed multimedia service provisioning. By default, the overall available bandwidth of HCCA channels is limited to $40 \%$ of the total available physical layer bandwidth. The 4 access categories (AC_VO, AC_VI, AC_BE and AC_BK) in EDCA provides differentiated channel access priority among different access categories, but if multiple channel accesses are requested for a same access category, contentions are generated, and guaranteed bandwidth provisioning is not possible.

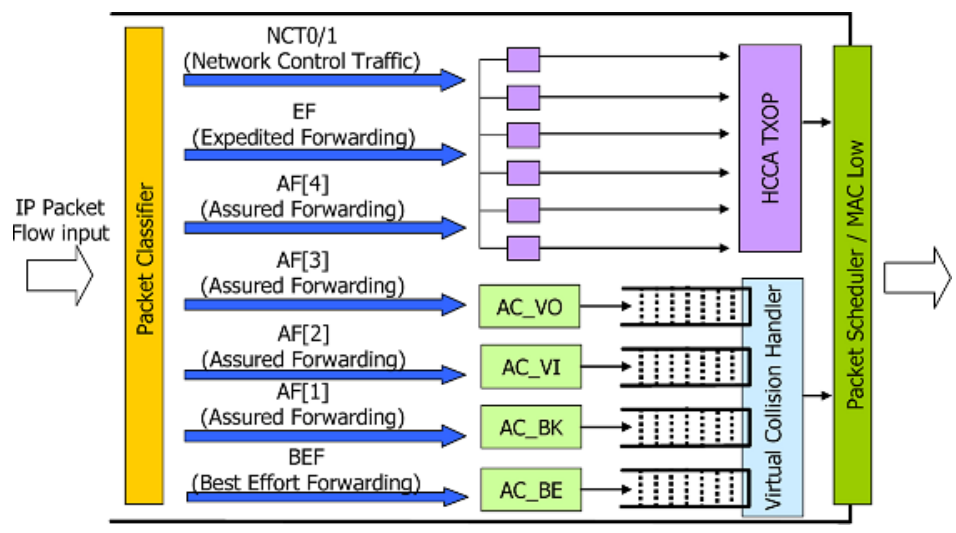

Fig. 2. Mapping between DiffServ class-types and IEEE 802.11e HCCA and EDCA channels

In the mapping scenarios between H.264 slice type and IEEE 802.11e HCCA and EDCA channels, we can consider several different alternatives, as shown in Table 2. In H.264 baseline profile encoding, only I and P slices are generated without B slices.

Table 2. Alternatives in mapping H.264 slice type and IEEE 802.11e HCCA and EDCA

\begin{tabular}{|l|l|l|l|l|}
\hline & \multicolumn{1}{c|}{ PSI } & \multicolumn{1}{c|}{ I } & \multicolumn{1}{c|}{ P } & \multicolumn{1}{c|}{ B } \\
\hline Case 1 $($ AC_VI only) & AC_VI & AC_VI & AC_VI & AC_VI \\
\hline Case 2 $($ AC_VO+AC_VI $)$ & AC_VO & AC_VO & AC_VI & AC_VI \\
\hline Case 3 $($ HCCA+ AC_VI $)$ & HCCA & HCCA & AC_VI & AC_VI \\
\hline Case 4 (AC_VO+AC_VI+AC_BE) & AC_VO & AC_VO & AC_VI & AC_BE \\
\hline Case 5 $($ HCCA+ AC_VI+AC_BE) & HCCA & HCCA & AC_VI & AC_BE \\
\hline
\end{tabular}


So, only case $1 \sim 3$ are applicable for H.264 baseline profile encoding for video phone and teleconference.

\section{Performance Analysis of Differentiated H.264 Video Transmission on IEEE 802.11e EDCA and HCCA Channels}

\subsection{Simulation Configuration}

In order to evaluate the differentiated QoS provisioning performance of IEEE 802.11e with HCCA and EDCA, we performed a series of ns-2 simulations. In our simulation, the QSTA are communicating through IEEE $802.11 \mathrm{~b}$ physical wireless links with $6 \mathrm{Mbps}$ physical transmission rate in infrastructure mode.

Currently, in the ns-2 network simulation configuration, each QSTA generates same H.264/AVC video traffic without internal virtual collision detection. To verify the performance of QoS provisioning, we measured the throughput, delay, jitter and packet loss rate. To generate network congestion situation, the number of active QSTAs has been gradually increased, and the performance parameters have been measured. At a certain level of increased traffic amount, contention occurs and severe packet drop and increased delay deteriorate the video quality.

\subsection{Analysis of the H.264 Extended Profile Video Transmissions}

Fig. 3, Fig. 4 and Fig. 5 depicts the throughput, packet delay and packet loss in the mapping scenarios for H.264 extended profile video transmission where I, P and B slices are transmitted.

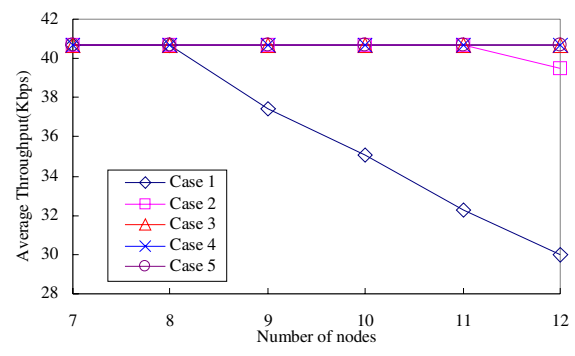

(a) Throughput of I slice type

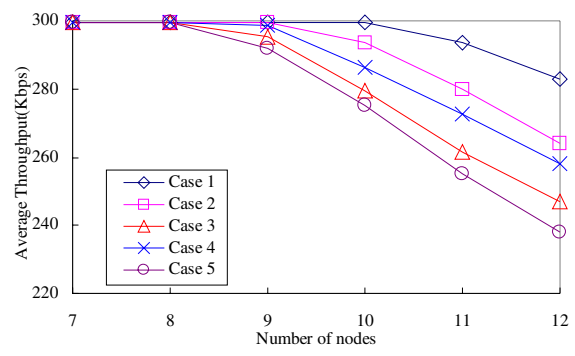

(b) Throughput of P slice type

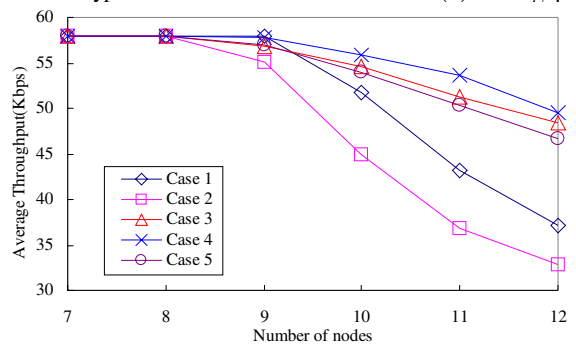

(c) Throughput of B slice type

Fig. 3. Throughput of I, P, B slice types from H.264 extended profile 
Fig. 3 compares the average throughputs of each slice type at different mapping scheme. In Fig. 3 (a), when I slices are transmitted via HCCA or AC_VO (Case 2 5 ), the throughput is guaranteed even when the number of node increases; when I slices are transmitted via AC_VI only (Case 1) competing with other P and B slices, the throughput is reduced as the number of node increases. These trends are also shown in Fig. 3 (b) where $\mathrm{P}$ slices are transmitted through AC_VI or AC_VO competing with other nodes or other slice types. In Fig. 3 (c), we can see that when B slices are transmitted through AC_BE competing with other slice types and nodes, its throughput is reduced seriously.

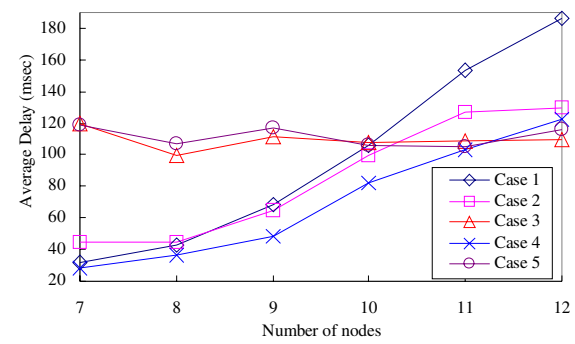

(a) Delay of I slice type

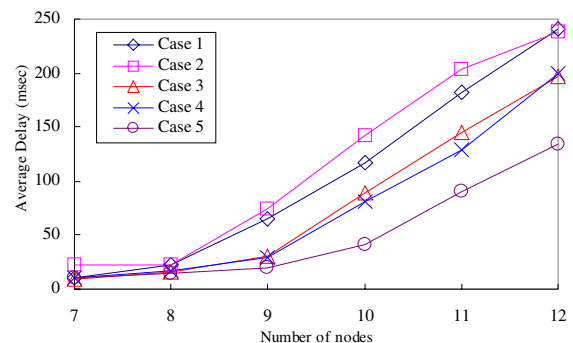

(b) Delay of P slice type

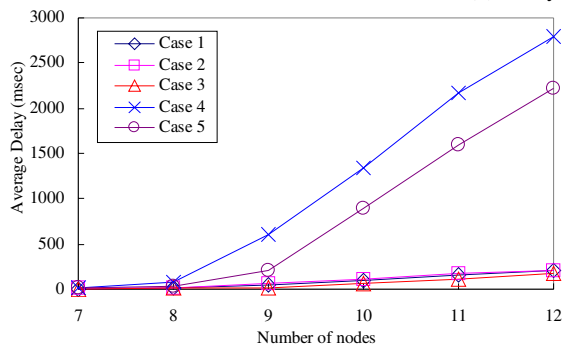

(c) Delay of B slice type

Fig. 4. Delay of I, P, B slices from H.264 extended profile

Fig. 4 compares the average end-to-end packet delays of each slice type from H.264 encoder with extended profile. From Fig. 4 (a), we can see that the delays of EDCA channels increase gradually as the number of nodes increases. But, the delays of HCCA channels are remained at almost the same value. Especially in Fig. 4 (c), the delay of B slices through AC_BE increases seriously beyond $500 \mathrm{~ms}$ when the number of node increases. Since the end-to-end delay for video telephony or video conference is requested to be less than $400 \mathrm{~ms}$, we can determine that any mapping schemes with AC_BE are not applicable for realtime conversational video telephony or video conference.

Fig. 5 compares the average packet loss ratios of each slice type at different mapping scheme. The packet loss of I slice type is increased when only AC_VI is used for all slice types because of the contention with P and B slices. Throughout the simulations, we found that H.264 decoding module in JM 10.2 reference software has severe difficulties when the packet loss ratio is more than $20 \%$. To solve this decoding problems, appropriate error concealment functions are needed. 


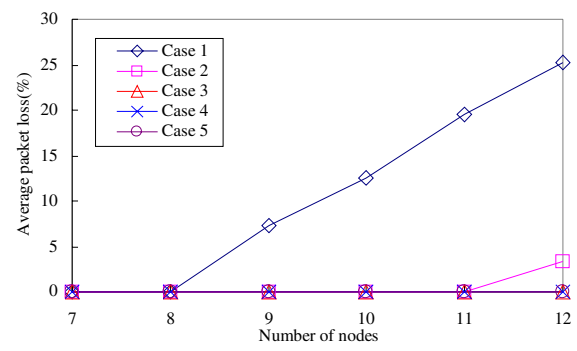

(a) Packet loss of I slice type

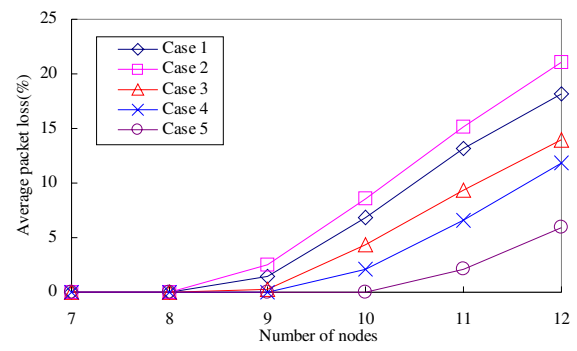

(b) Packet loss of P slice type

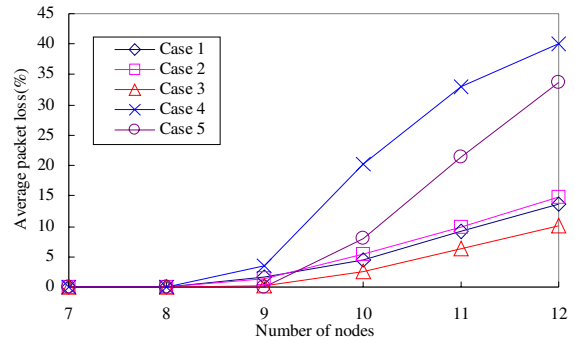

(c) Packet loss of B slice type

Fig. 5. Packet loss of I, P, B slices from H.264 extended profile

Fig. 6 depicts the comparison of average PSNRs of each mapping scheme. The mapping schemes of using Case 1(AC_VI only) and Case 4(AC_VO+ AC_VI+AC_BE) are showing severely deteriorated PSNR less than $30 \mathrm{~dB}$ when the number of nodes is increased beyond 11. But, the mapping scheme that uses HCCA for I slices, AC_VI for P slices, and AC_BE for B slices (Case 5) maintains the acceptable value of PSNR according to the number of node increases, because high priority packets (I and P slice packets) experience less packet loss than other schemes. In Case 5, packet loss rate of B slice type is more severe but less influence on PSNR as previously mentioned in chapter 3.1 (Fig. 1).

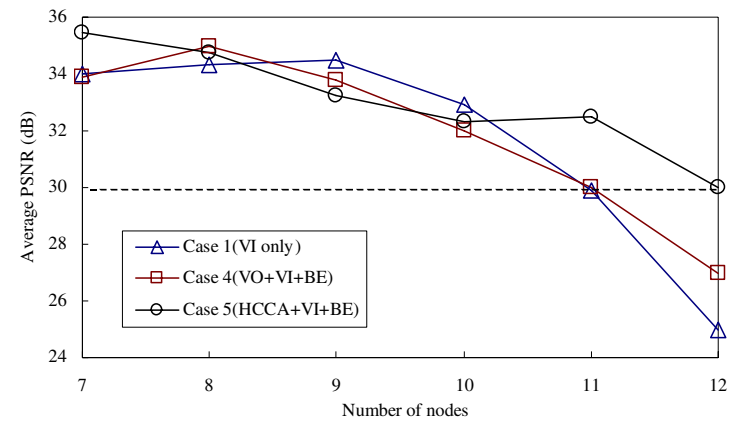

Fig. 6. Comparisons of PSNRs of mapping schemes for H.264 extended profile 
From the simulation results of Fig. $3 \sim$ Fig. 6, we can find the mapping schemes of using AC_BE cannot provide good QoS performance for realtime multimedia telephony or teleconference services. We also simulated using Paris CIF video sequence in extended profile, that has longer playing time than Foreman, and got the similar results in the average PSNR. In next section 4.3, we analyze H.264 baseline profile video transmissions for realtime multimedia telephony and teleconference in detail.

\subsection{Analysis of the H.264 Baseline Profile Video Transmissions}

H.264 baseline profile has been standardized for realtime video telephony or video teleconference where end-to-end delay is required to be limited within $400 \mathrm{~ms}$ for realtime conversational quality. In H.264 baseline profile, only I slices and P slices are generated. Fig. $7 \sim$ Fig. 10 compare the possible mapping schemes for realtime H.264 baseline profile video transmission on IEEE 802.11e wireless access network.

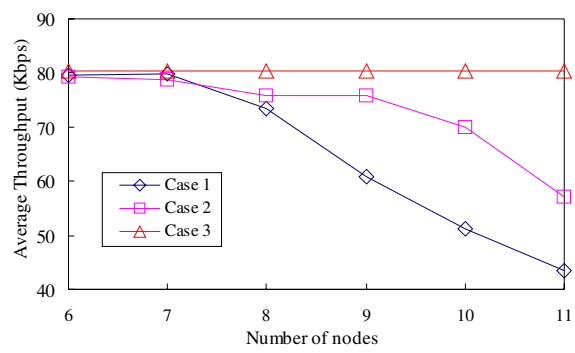

(a) Throughput of I slice type

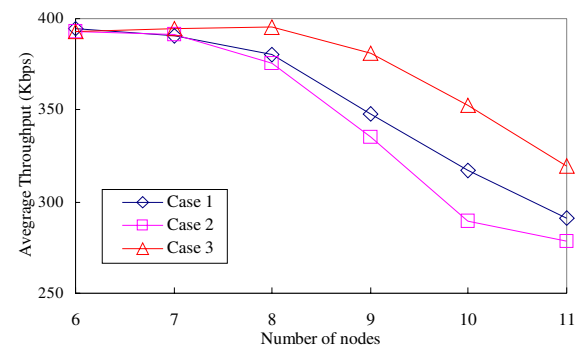

(b) Throughput of P slice type

Fig. 7. Throughput of I and P slices from H.264 baseline profile

In Fig. 7, Fig. 8 and Fig. 9, we can see that the mapping scheme of HCCA for I slices and EDCA AC_VI for P slices (Case 3) provides the best performance in guaranteed throughput, delay and packet loss ratio. The delays are within the limit of $400 \mathrm{~ms}$ in our simulation results, which are reasonable for realtime video teleconference in IEEE 802.11e.

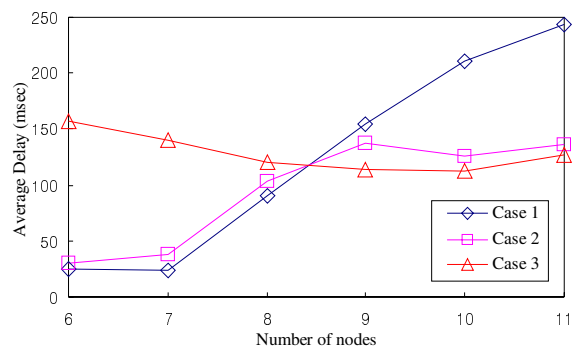

(a) Delay of I slice type

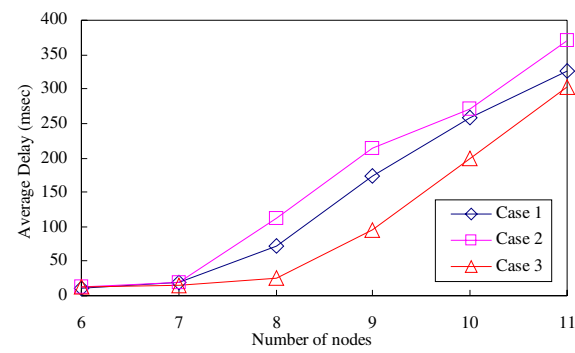

(b) Delay of P slice type

Fig. 8. Delay of I and P slices from H.264 baseline profile 


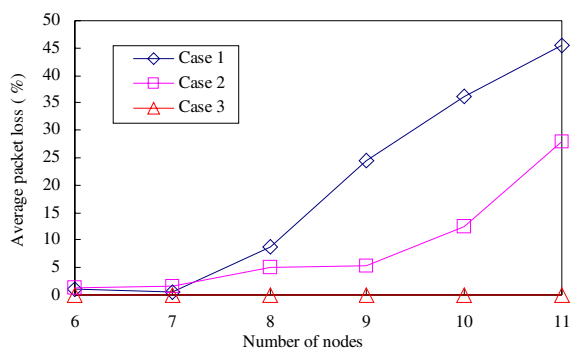

(a) Packet loss of I slice type

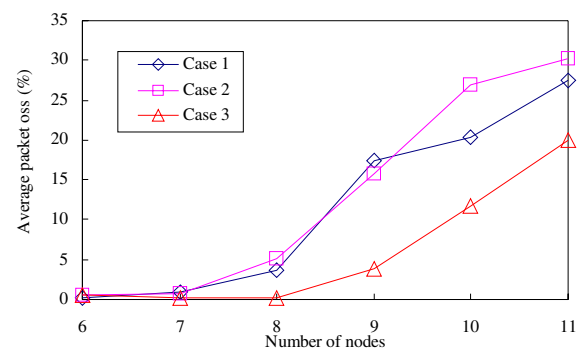

(b) Packet loss of P slice type

Fig. 9. Packet loss of I and P slices from H.264 baseline profile

As shown in Fig. 9 (a), Case 1 shows increased packet loss (beyond $25 \%$ ) when the number of nodes is beyond 10, and the H.264 decoding at JM 1.0 was not possible. There are no packet losses in Case 3 because HCCA reserves sufficient bandwidth through TSPEC negotiation in connection setup time similar in the previous simulation with extended profile.

Fig. 10 compares the PSNRs of mapping schemes for $\mathrm{H} .264$ baseline profile for realtime video teleconference. The $\mathrm{H} .264$ video transmission using Case 1 (AC_VI only) and Case 2 (AC_VO+AC_VI) show poor performance in PSNR when the number of node increases beyond 8 .

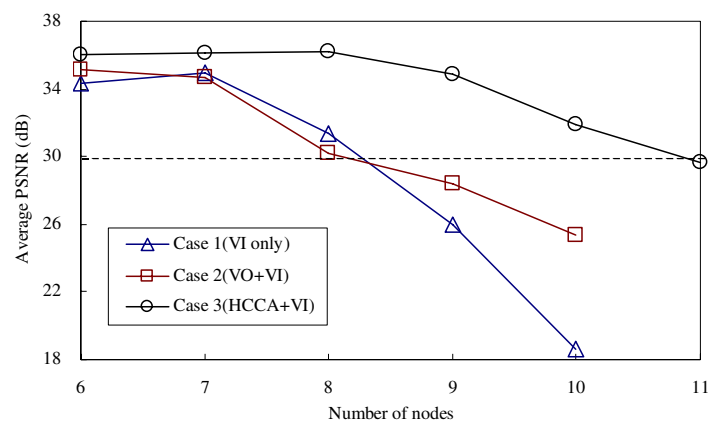

Fig. 10. Comparison of PSNRs of mapping schemes for H.264 baseline profile

From the various simulation analysis on the mapping scheme of H.264 baseline profile on IEEE 802.11e HCCA and EDCA channels for realtime video teleconference application, we can find that the mapping scheme of HCCA for I slices and EDCA AC_VI for P slices of H.264 baseline profile provides the best performance in guaranteed bandwidth provisioning, delay, packet loss and overall PSNR for realtime video teleconference. We also simulated using Paris CIF video sequence in baseline profile, and got the similar results in the average PSNR. 


\section{Conclusions}

In this paper we analyzed the management of IEEE 802.11e WLAN for realtime QoSguaranteed teleconference services with differentiated H.264 video transmission. We performed a series of simulations to compare the channel allocation schemes of IEEE 802.11e Wireless LAN to transmit I, P and B slices from H.264 encoder using different channels of both HCF controlled channel access (HCCA) and enhanced distributed channel access (EDCA). We compared the different mapping scenarios, and analyze the QoS provisioning performance for $\mathrm{H} .264$ baseline profile based realtime multimedia teleconference service where $400 \mathrm{~ms}$ delay time limit is required. From the simulations, we found that the mapping scheme of HCCA for I slices and EDCA AC_VI for P slices of H.264 baseline profile provides the best performance in guaranteed bandwidth provisioning, delay (less than $400 \mathrm{~ms}$ ), packet loss and overall PSNR for realtime video teleconference.

\section{References}

1. IEEE Standard for Information Technology, Local and metropolitan area networks - Part 11: Wireless Medium Access Control (MAC) and Physical Layer (PHY) specifications, Amendment 7: Medium Access Control (MAC) Quality of Service (QoS) Enhancements, IEEE std 802.11e/D12.0, 2004.

2. Qiang Ni, "Performance Analysis and Enhancements for IEEE 802.11e Wireless Networks," IEEE Network, July/August 2005, pp. $21 \sim 27$.

3. Stefan Mangold, Sunghyun Choi, Guido Hiertz, Ole Klein, and Bernhard Walke, "Analysis of IEEE 802.11e for QoS support in Wireless LANs," IEEE Wireless Communications, December 2003, pp. $40 \sim 50$.

4. Ramos, N., Panigrahi, D. Dey, S., Quality of service provisioning in 802.11e networks: challenges, approaches, and future directions, IEEE Network, Vol. 19, July-Aug 2005.

5. ITU-T Recommendation H.264, Advanced video coding for generic audiovisual services, March 2005.

6. Wiegand, T., Sullivan, G. J., Bjntegaard, G., Luthra, A, Overview of the H.264/AVC video coding standard, IEEE Transactions on Circuits and Systems for Video Technology, Vol. 13, July 2003, ppl 560-576.

7. Ostermann, J., et. al., Video coding with H.264/AVC: tools, performance, and complexity, IEEE Circuits and Systems Magazine, Vol. 4, First Quarter 2004, pp. 7-28.

8. Iain E. G. Richardson, H.264 and MPEG-4 Video Compression - Video Coding for Next Generation Multimedia, John Wiley \& Sons, 2003.

9. Adlen Ksentini, Mohamed Naimi, Abdelhak Gueroui, "Toward an improvement of H.264 video transmission over IEEE 802.11e through a cross-layer architecture," IEEE Comm. Mag., January 2006, pp. 107-114.

10. Deyun Gao, Jianfei Cai, Bao, P., Zhihai He, MPEG-4 video streaming quality evaluation in IEEE 802.11e WLANs, ICIP 2005, Vol. 1, Sept. 2005, pp. 197-200.

11. Yang Xiao, Voice and Video Transmissions with Global Data Parameter Control for the IEEE 802.11e Enhance Distributed Channel Access, IEEE Transactions on Parallel and Distributed Systems, Vol. 15, No. 11, Nov. 2004, pp. 1041-1053.

12. S. Wenger, M.M. Hannuksela, T. Stockhammer, M. Westerlund, D. Singer, RTP Payload Format for H.264 Video, Internet proposed standard RFC 3984, February 2005.

13. JM 10.2 reference software, http://iphome.hhi.de/suehring/tml/. 